\title{
Niños y adolescentes 1 agresivos
}

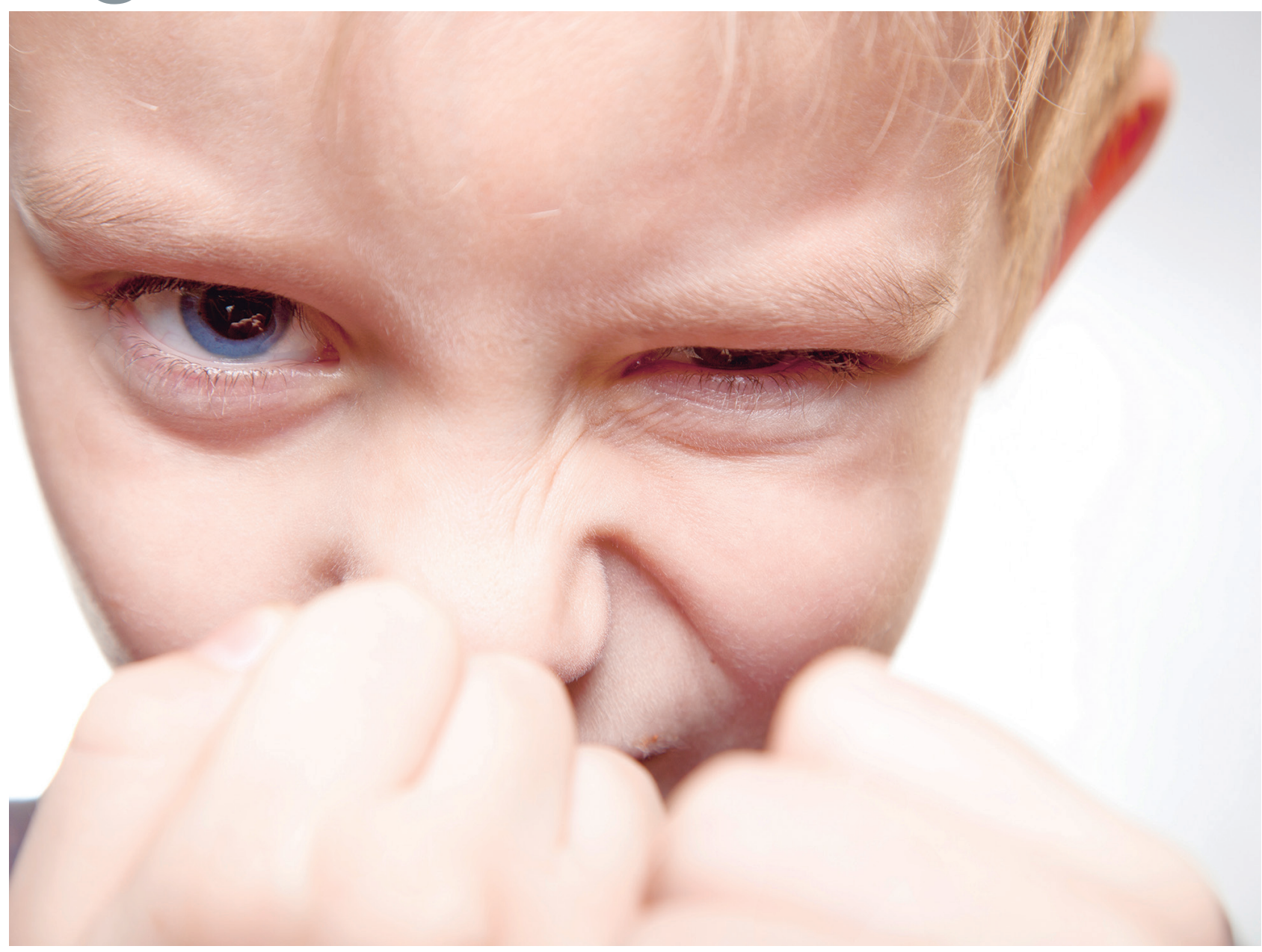

El artículo describe la agresividad en niños y adolescentes analizando su conceptualización, diferente tipología y manifestaciones, evaluación y modelos teóricos que nos permiten comprender por qué nuestros

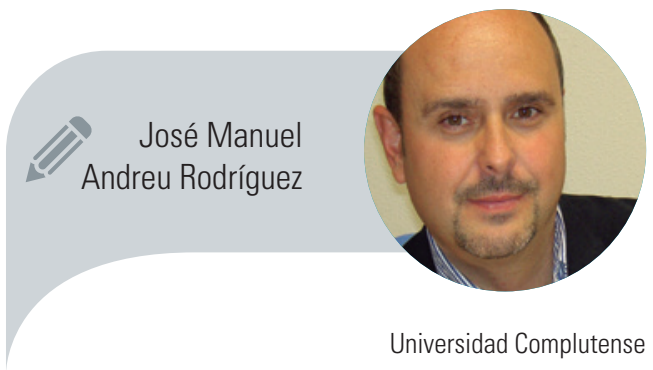
hijos pueden llegar a ser agresivos. Desde jmandreu@ucm.es estos planteamientos, se llega a una serie de reflexiones sobre diferentes estrategias que permiten reducir y controlar la agresividad infantil en contextos escolares y familiares. 


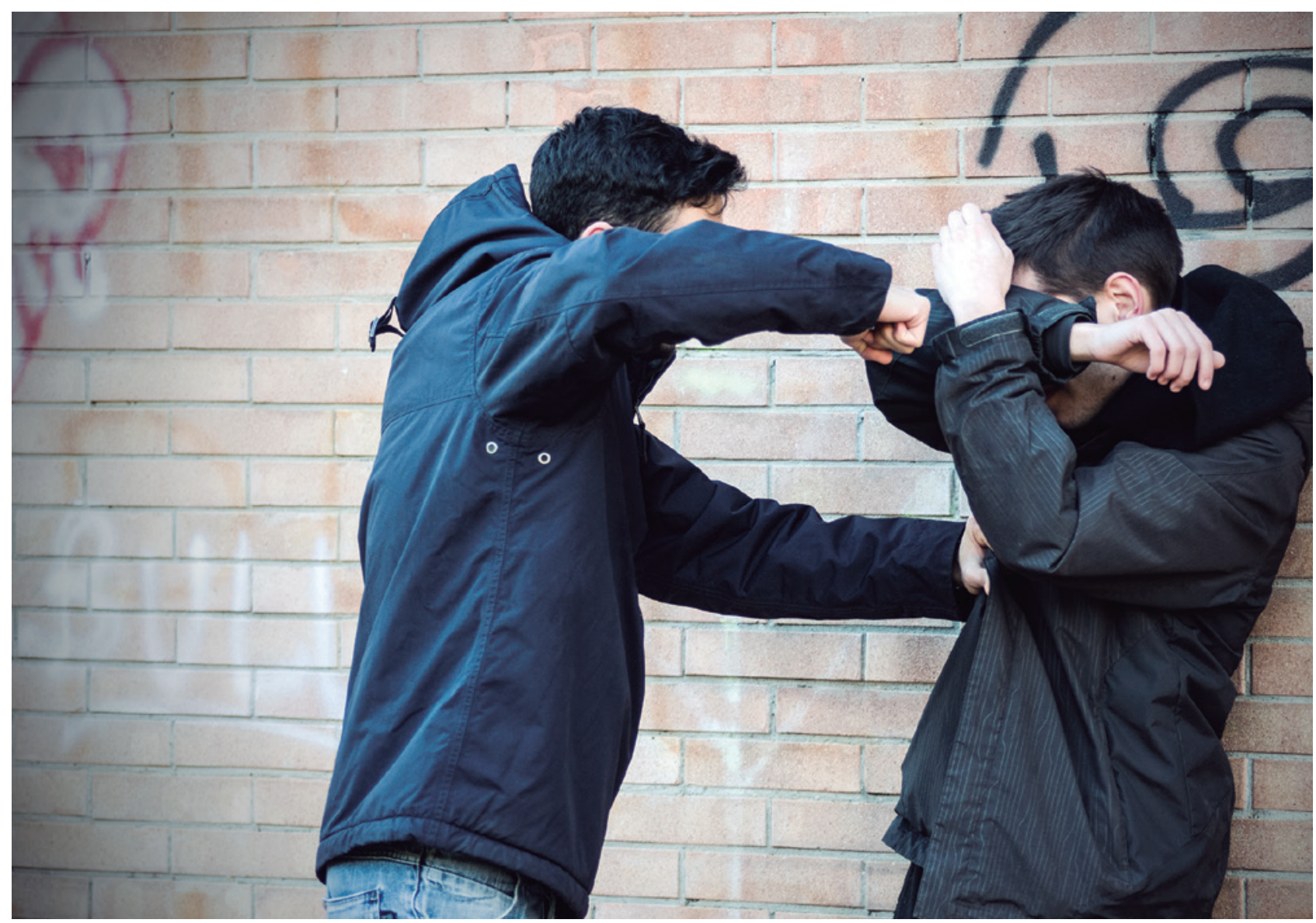

\section{¿Qué es la agresividad?}

En primer lugar, sería necesario aclarar que la agresividad en niños y adolescentes es, por lo general, resultado de un proceso psicológico que implica la acción conjunta de múltiples pensamientos, emociones y conductas. Esto refleja la multidimensionalidad de este comportamiento por la cual diferentes mecanismos psicológicos se combinan mentalmente para crear distintas formas de conducta agresiva. Así, la agresividad estaría en función de una serie de objetivos, propósitos y motivaciones, por lo que, en otras palabras, ningún comportamiento agresivo se realiza sin alguna motivación. Consecuentemente, el primer paso para comprender por qué nuestros hijos son agresivos, es preguntarse cuál o cuáles son los motivos que subyacen en dicho comportamiento.

Es importante comprender que en la infancia son normales los ataques de agresividad o de conductas agresivas que puedan causar daño. Conductas como pegar a otros, ofenderlos, burlarse de ellos o tener rabietas suelen formar parte de la agresividad infantil. Cuando algunos niños persisten en su conducta y no son capaces de controlar su fuerte genio, pueden sentirse frustrados provocando sufrimiento y rechazo en los demás. El comportamiento agresivo del niño es normal en este contexto, sin embargo, el problema al que nos enfrentamos es cómo enseñar a nuestros hijos a saber controlarlo a tiempo. Y aquí es precisamente donde actuamos los adultos.

Profundizando en la agresividad infantil, ésta puede venir acompañada de acciones que están dirigidas a controlar, manipular o dañar seriamente a los demás. Este tipo de agresividad ya no posee utilidad alguna para el proceso de adaptación social, sino que es básicamente aprendida a lo largo del desarrollo y de las experiencias individuales de cada niño y adolescente. Esta agresión disfuncional tendría un marcado carácter intencional con el objetivo de conseguir fines u objetivos más allá de la defensa propia y la supervivencia. Así, los niños y adolescentes más agresivos llegarían a inhibir sus sentimientos de culpa, remordimientos o empatía, transgrediendo normas y provocando daño a quienes consideran su oponente. A este tipo de adolescentes les afectan desproporcionadamente las injusticias, la pérdida de su estatus, 
Figura 1. Formas de expresión de la agresividad

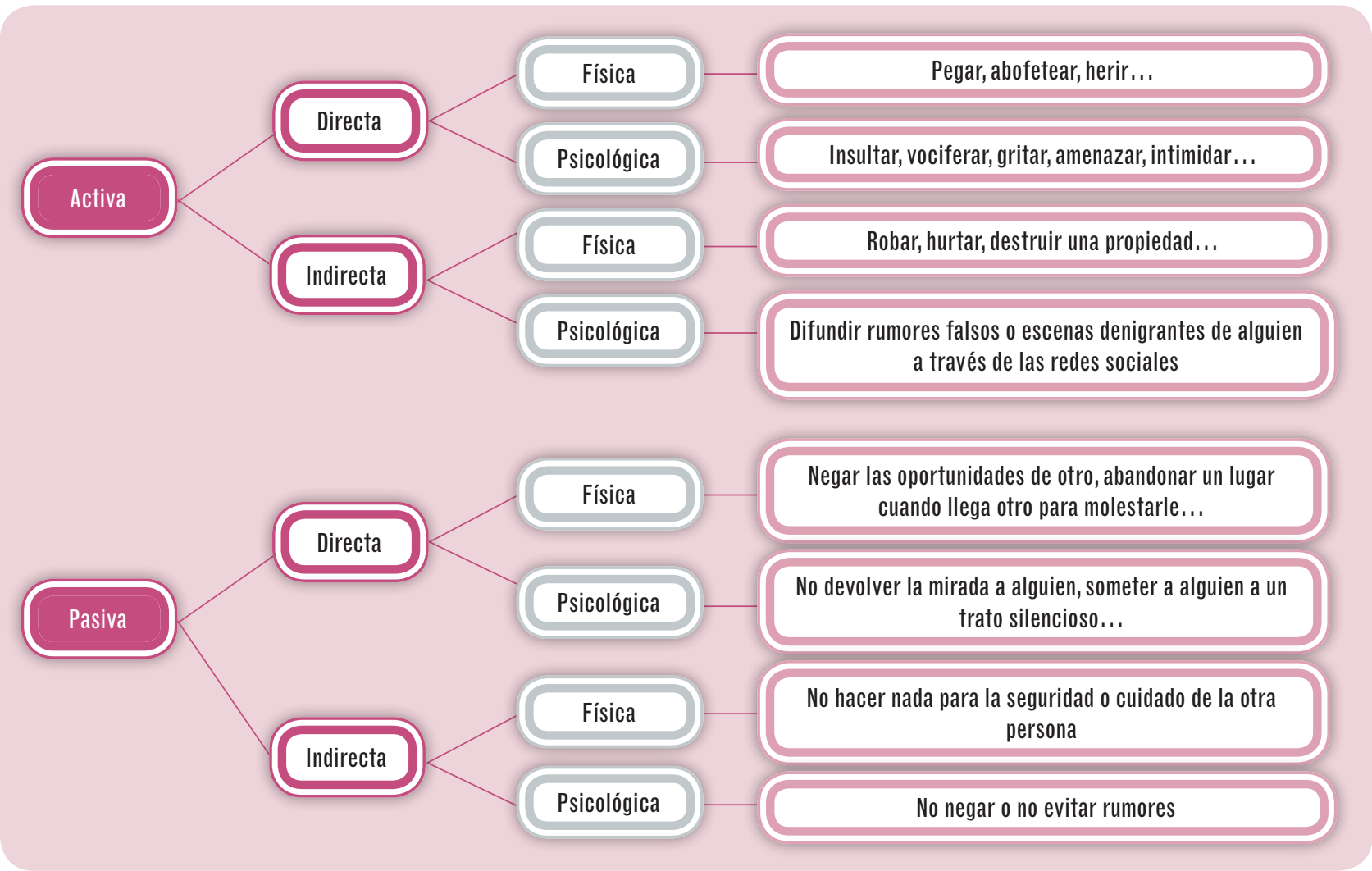

de su dominio personal o incluso la puesta en duda de su eficacia personal.

\section{¿Cómo se manifiesta la agresividad?}

Es importante tener en cuenta que la agresividad durante este periodo evolutivo se manifiesta de múltiples y variadas formas en función de distintos niveles: 1) a nivel físico, cuando se busca hacer daño o herir a alguien a través de una acción física; y 2) a nivel psicológico, cuando se pretende herir o lastimar al otro mediante amenazas, insultos o menosprecios. Estos dos tipos de agresión se suelen expresar de forma directa, cara a cara, pero también pueden tomar otros cauces para dañar indirectamente o de forma relacional a la otra persona (p. ej., cuando se daña la reputación e intimidad de un compañero a través de las redes sociales). Estos tipos de expresión no directa en las que se expresa la agresividad a través de otros medios o mecanismos son menos visibles, pero no por ello menos lesivos o dañinos para el ofendido. Y es importante darnos cuenta a tiempo. La figura 1 describe las distintas formas
La agresividad estaría en función de una serie

de objetivos, propósitos y molivaciones, por lo

que ningún comportamiento agresivo se realiza

sin alguna molivación

de expresión que puede tomar la agresividad durante la infancia y adolescencia.

Ya se ha hecho mención con anterioridad a que la agresividad, además de ser desencadenada por diferentes procesos psicológicos, surge a través de la acción de una serie de motivaciones que impelen, desencadenan o dirigen la conducta con una intención u objetivo determinado. Hay que tener en cuenta que un niño o un adolescente que agrede a otro, puede tener múltiples y diferentes motivos. Teniendo en cuenta esta dimensión motivacional, se ha distinguido precisamente entre agresión instrumental (premeditada) y expresiva (impulsiva) para reflejar que la agresión 
Siguiendo las consideraciones precedentes es posible identificar una serie de estrategias generales que permiten ayudar a afrontar las conductas agresivas en el aula.

1. Identificar el tipo de conducta agresiva. La agresividad se puede manifestar de muchas maneras: pataleos o gritos, agresiones físicas a los padres, compañeros 0 profesores. Es importante que identifiquemos con la mayor precisión posible el tipo de conducta agresiva. Cuándo se produce y por qué, ir anotando los episodios de agresividad que tiene el niño, qué los desencadena, qué los mantiene y cuál es la reacción en el contexto.

2. Objetivos para modificar la conducta. Una vez que se han identificado los motivos que causan la conducta agresiva y cómo se desarrolla, se deben plantear los objetivos que queremos alcanzar. Si por ejemplo hemos comprobado que el niño suele ser agresivo en el patio del colegio, y que generalmente lo es para conseguir algo por parte de sus compañeros, sería necesario contar con un profesor que esté pendiente del juego en el que está participando este niño para regularlo.

3. Establecer normas y límites desde el principio. Si los alumnos saben desde el principio del año las consecuencias de sus acciones, será mucho más fácil controlar el comportamiento de los alumnos en el aula. Es necesario explicar a los alumnos las consecuencias de determinadas acciones negativas y las consecuencias de determinadas acciones positivas.

4. Evitar el castigo. Es importante conocer diferentes técnicas de modificación de la conducta para aplicarlas en el contexto escolar que son de especial valor para reducir las conductas agresivas en niños y adolescentes. Por ejemplo, el tiempo fuera (retirando las condiciones del medio que permiten obtener algún reforzador positivo) y el coste de respuesta (retirando algún reforzador positivo de manera contingente a la emisión de una conducta agresiva).

5. Estar preparados para el comportamiento agresivo. Hay muchas señales que nos pueden indicar que un comportamiento agresivo está a punto de producirse en el niño (ponerse rojo, emitir sonidos, tirar cosas al suelo, patalear... .). Si no se ha podido prevenir esa situación, se debe estar preparado para afrontarla con la mayor serenidad posible.

6. Intervenir de forma precoz. Es fundamental identificar e intervenir sobre la conducta agresiva lo antes posible. En caso contrario, estas conductas cada vez irán de mal en peor y llegará un momento en el que puedan llegar a ser difícilmente controlables. En este punto, es importante contar con la ayuda de un profesional.

puede utilizarse con distintos objetivos, como por ejemplo como una estrategia de acción intencionada y finalista cuya principal motivación no sólo es la de producir daño, dolor o perjuicio, sino por el contrario, la de coaccionar, dominar, resolver conflictos interpersonales y obtener así cualquier tipo de beneficio personal (poder, dominio, reputación y autoestima).

\section{¿Cómo evaluar la agresividad?}

La identificación y evaluación de la agresividad infantil es una tarea compleja que requiere recopilar información acerca de sus parámetros (intensidad, frecuencia y duración), de sus condi- cionantes (factores desencadenantes y mantenedores), de su contexto (educativo, familiar y social) y tipología (en función de su naturaleza y motivación). Para facilitar este proceso de recopilación de información se cuenta con la ayuda de instrumentos estandarizados tales como cuestionarios, escalas y test psicológicos. En nuestro país contamos con el Cuestionario de Agresividad Premeditada e Impulsiva (ANDREU, 2010), editado en TEA Ediciones, que permite precisamente la evaluación específica de diferentes tipos de agresividad. Este instrumento identifica el perfil característico de agresividad a partir del cual es posible obtener pautas dirigidas a la intervención educativa y psicológica a realizar con el niño o el adolescente problemático.

\section{¿Cómo se aprende a ser agresivo?}

Albert Bandura fue el primer autor que planteó que la conducta agresiva infantil es un comportamiento que se adquiere mediante los mismos procesos de aprendizaje que cualquier otra conducta social. En sus primeras investigaciones, pudo comprobar cómo los niños pueden imitar rápidamente la conducta de un modelo adulto agresivo. Este aprendizaje observacional de la conducta agresiva sucede fundamentalmente dentro de la familia, por lo que Bandura postuló que el comportamiento de los niños que es reforzado por los padres o los adultos que rodean al niño es el que tiene mayor tendencia a repetirse en el futuro.

En este proceso de aprendizaje por observación intervienen cuatro procesos: procesos atencionales, de retención, de reproducción y de motivación, que serían los factores condicionantes de la conducta agresiva. De esta forma, un adolescente puede responder de forma agresiva al prestar atención al comportamiento del modelo, al codificar esa información en la memoria a largo plazo, si tiene las capacidades para reproducir esas conductas de forma motora y, finalmente, si espera obtener refuerzos positivos después de realizarlas. Los niños y adolescentes retienen mejor lo que han aprendido cuan- 


\section{La conducta agresiva infantil es un comportamiento que se adquiere mediante los mismos procesos de aprendizaje que cualquier otra conducta social}

do prevén que pueden obtener beneficios o consecuencias positivas de ello porque esto les motiva a repetir la conducta del modelo agresivo. En consecuencia, es muy importante que los padres o los adultos que rodean al menor, especialmente cuando éstos son difíciles de educar, no discutan o sean agresivos en su presencia puesto que acabarían aprendiendo este comportamiento por imitación.

\section{Lo que piensan los niños influye en su agresividad}

Kenneth Dodge sostiene que los niños y adolescentes con problemas de conducta agresiva, afrontan las situaciones sociales con una serie de capacidades psicológicas limitadas al tener interiorizadas una serie

\section{CAMIInARDO JUחTOS}

Se ofrecen aquí algunas sugerencias prácticas para padres que puedan servir de ayuda para prevenir y facilitar que las conductas agresivas disruptivas no aparezcan durante el desarrollo social de nuestros hijos:

1. Es necesario que los padres enseñen a los niños a ponerse en el lugar del otro. A partir de los dos años de edad, los niños ya tienen más conciencia del otro y de conductas como compartir, colaborar y ayudar.

2. Es muy importante la comunicación emocional entre padres e hijos. Esta comunicación fomenta la confianza de los niños y la transmisión de valores por parte de los padres. Y debe de acompañarse de un adecuado reconocimiento de las conductas positivas (no agresivas) que emite el niño 0 adolescente.

3. Aprender a ser solidarios es un elemento clave. La solidaridad debe ser enseñada y transmitida a los niños a través de nuestro ejemplo. Los padres deben ejercitar la solidaridad con ellos y con los demás.

4. Es recomendable que los padres hablen a sus hijos de lo que está bien y mal, y que pongan límites y normas de forma consistente. No se deben de aplicar castigos pero sí contar con la ayuda de técnicas para reducir la agresividad en el contexto familiar (tiempo fuera 0 coste de respuesta).

5. Una educación permisiva 0 autoritaria no es una educación adecuada para un buen funcionamiento de la dinámica familiar. Para promover límites, normas y valores es necesario que el niño reciba una educación en un entorno familiar positivo.

6. A veces los padres no somos cuidadosos en lo que les decimos 0 en lo que hacemos.Y nos pueden escuchar hablando mal de otra persona, 0 gritando mientras conducimos. Nuestro comportamiento es su mejor educación.
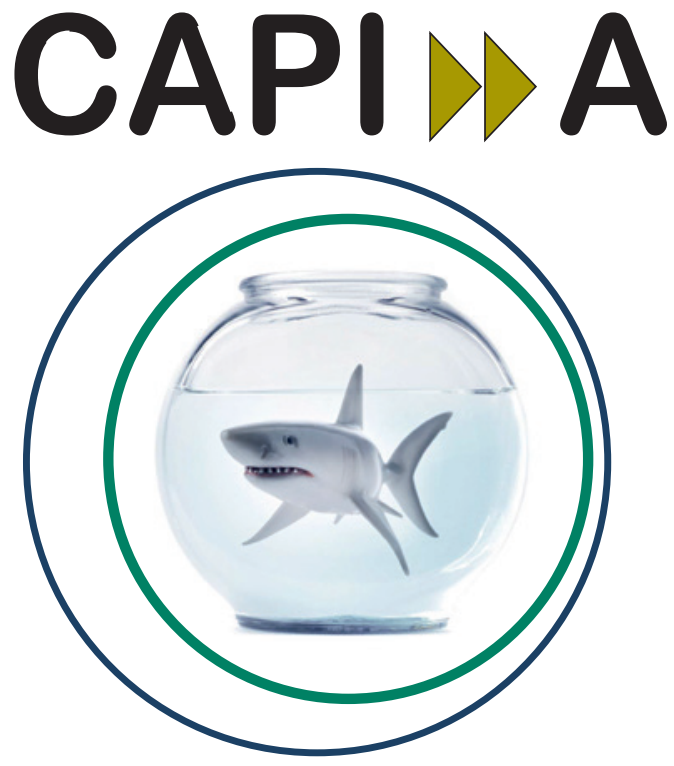

A la vanguardia de la evaluación psicológica www.teaediciones.com

\section{Cuestionario de Agresividad Premeditada e Impulsiva en Adolescentes}

Evalúa y distingue entre la agresividad premeditada, para conseguir un objetivo y la agresividad impulsiva, como respuesta a una amenaza en adolescentes, tanto en población general como clínica.

Clave para la evaluación funcional de la agresividad y el diseño de programas de prevención e intervención en función de los resultados obtenidos en el instrumento. 


\section{IIII. ÁQORA DE DROFESORES}

1. Es esencial que en la escuela se trabaje la empatía en los niños y adolescentes. En otras palabras, que se trabaje de forma transversal el despertar de la preocupación por los demás.

2. Cuando los alumnos ayuden a sus compañeros, llevan a cabo juegos cooperativos, 0 se comporten de forma solidaria y positiva con los demás, es importante recompensarles y mostrarles que estamos orgullosos de su actitud.

3. Es fundamental que los objetivos que se van a trabajar en el colegio tengan continuidad en el ámbito familiar. La educación de nuestros hijos implica una acción coordinada en ambos contextos.

4. Una comunicación efectiva entre los adultos que forman parte de la vida del niño es necesaria para cumplir con nuestro principal objetivo: comprender y reducir la expresión de la agresión en el entorno escolar y familiar.
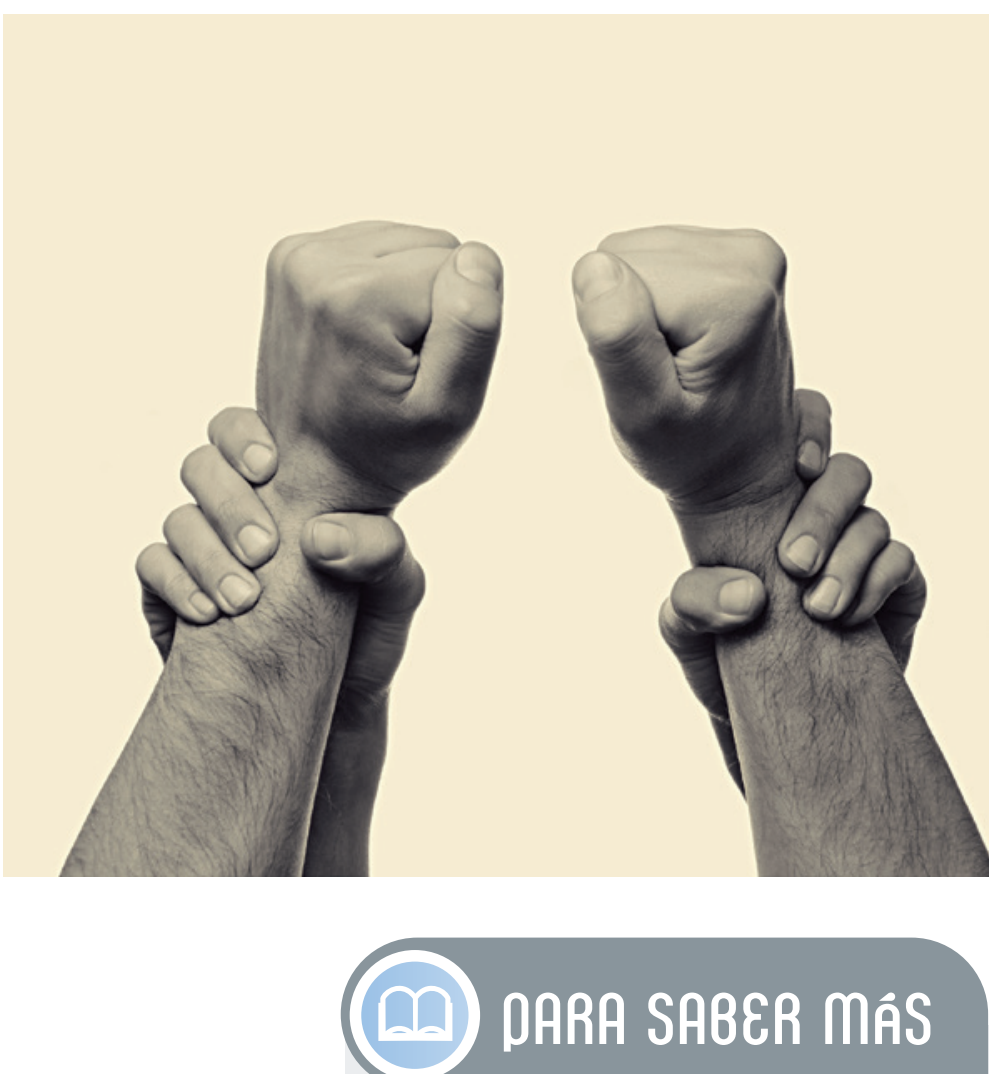

Aguilar, A. S., Andreu, J. M. y Peña, M. E. (2012). Prevención del maltrato en la escuela: experiencia de un programa entre alumnos de educación secundaria. San Salvador: Ediciones de la Universidad Tecnológica de El Salvador.

AndREu, J. M. (2010). Cuestionario de agresividad premeditada e impulsiva en adolescentes (CAPI-A). Madrid: TEA Ediciones.

Andreu, J. M., Peña, M. E. y Penado, M. (2012). Agresión juvenil: una investigación empírica sobre sus causas. Madrid: Editorial Académica Española. actos accidentales o ambiguos en función de las intenciones de unos modelos que eran observados a través de un televisor. A raíz de los resultados obtenidos en estos estudios, los autores afirmaron que cuando un niño se enfrenta a una situación social novedosa, éste realiza una serie de pasos hasta que se produce la respuesta agresiva: (1) el niño codifica las señales internas y externas; (2) representa e interpreta esas señales; (3) busca mentalmente las posibles respuestas a la situación; y (4) selecciona la respuesta.

Este modelo sostiene que durante los pasos de codificación e interpretación de los indicios sociales, los niños focalizan y codifican algunos indicios en particular $y$, posteriormente, tomando como base esos indicios, construyen una interpretación mental de la situación. Durante los siguientes pasos, los niños formulan metas u objetivos para resolver el incidente, generan estrategias de solución de problemas, evalúan la probable efectividad de las estrategias y seleccionan una respuesta para finalmente emitir una respuesta al daño percibido. Los autores sostienen que algunos tipos de procesamiento ineficaz son los responsables de que los niños se comporten agresivamente en respuesta a un conflicto o a provocaciones por parte de sus compañeros. Si un niño interpreta como hostil el daño que se produce en circunstancias ambiguas, éste tendrá la tendencia a considerar que este daño deriva de un intento hostil por parte de quien lo ha provocado, justificará su sentimiento de enfado y, por tanto, la utilización de una conducta agresiva •

\section{(-) \\ Agresividad; niños y adolescentes; contexto educativo; familia.}

Este artículo fue solicitado por PADRES Y MAESTROS en marzo de 2017, revisado y aceptado en junio de 2017. 